\title{
Preferencias de Elección de Escuela en Dos Casos de Alta Segregación Escolar
}

\author{
School Choice Preferences in Two High School Segregation \\ Cases
}

\author{
Claudia Córdoba * \\ Alonso Laborda \\ Claudia Reyes
}

Universidad de Chile, Chile

\begin{abstract}
Latinoamérica es la región con más altos niveles de desigualdad a nivel mundial, lo que se ve reflejado en sus sistemas escolares. En este escenario el caso chileno se destaca por un alto grado de segregación escolar lo que ha sido vinculado con la aplicación sistemática de políticas de mercado. Este fenómeno se relaciona con diferentes factores, siendo uno de ellos las valoraciones, estrategias y acciones que las familias despliegan en la elección de escuela. Este trabajo analiza discursos de elección de familias en dos casos, cada uno de ellos conformados por tres escuelas vecinas de distinto tipo (municipal, privada subvencionada y privada no subvencionada), existiendo entre ellas contrastes notables en cuanto a las características socioeconómicas de su estudiantado. Los casos evidencian, por tanto, la existencia de segregación escolar a una "microescala" que no ha sido abordada en estudios previos. La metodología utilizada es de tipo cualitativa. La producción de datos se efectuó mediante entrevistas semiestructuradas con 54 madres o padres de 6 establecimientos educativos. Los resultados muestran que la "libre elección" de escuela está limitada por los recursos económicos, lo que restringe las opciones en el "mercado escolar". Asimismo, se corrobora la existencia de elementos socioculturales que condicionan el proceso de elección de las familias de acuerdo con su nivel socioeconómico y otras características. Se concluye que el proceso de elección de escuela es un factor que produce y reproduce el fenómeno de la segregación escolar.
\end{abstract}

Descriptores: Segregación escolar; Escuela primaria; Elección de la escuela; Calidad de la educación; Zona urbana.

Latin America is the region with the highest levels of inequality, a fact that is reflected in their school systems. In the frame of a systematic application of market policies, the high levels of school segregation in Chile are highlighted. This phenomenon is related to different factors, one of them being the behavior of the families when choosing a school. This study analyzes the families' decision speeches in two cases, formed by three different types of nearby schools -municipal, private subsidized and private non-subsidized- where there are significant differences in the socioeconomic composition of the student body. The cases show, therefore, the existence of school segregation at a "micro-level". The methodology of the study is qualitative. The data was obtained by semi structured interviews of 54 mothers or fathers. The results show that the "free choice" of schools is limited by the economic resources, which restricts the options in the "school market". Likewise, the existence of socio-cultural elements determining the selection process of the families according to their socioeconomic level and other factors was confirmed. It is concluded that the process of school choice is a factor that produces school segregation.

Keywords: School segregation; Primary schools; Educational choice; Educational quality; Urban areas.

*Contacto: claudia.cordoba.c@usach.cl

ISSN: $1696-4713$

www.rinace.net/reice/

revistas.uam.es/reice
Recibido: $\quad 15$ de abril 2020

$1^{\text {a }}$ Evaluación: 13 de junio 2020

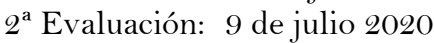

Aceptado: $\quad 6$ de agosto 2020 


\section{Introducción}

Latinoamérica es la región que a nivel mundial exhibe mayores grados de inequidad con brechas notables en las condiciones de los sectores más y menos acomodados (CEPAL, 2018). Esta problemática se refleja en sus sistemas educativos caracterizados por intensos niveles de segregación (Murillo, 2016; Murillo y Martínez-Garrido, 2017; Murillo et al., 2018), problema que además constituye uno de los principales tópicos de investigación educativa en la región (Murillo y Martínez-Garrido, 2019). Dentro de este escenario destaca el caso chileno como uno de los sistemas con más bajo grado de integración socioeconómica y académica (OCDE, 2019).

La segregación escolar alude a la disímil distribución de estudiantes entre escuelas en función de características tales como su nivel socioeconómico, pertenencia a grupos étnicos o minoritarios, condición de nativo o migrante o rendimiento académico (Murillo, Belavi y Pinilla, 2018). Se trata de un fenómeno complejo en el que confluyen variables estructurales y culturales, que Bellei (2013) organiza en tres categorías: contextuales, institucionales y socioculturales. Los factores contextuales tienen relación con el escenario en que se enmarcan los sistemas, siendo relevante la segregación residencial y otros fenómenos: focos de pobreza, olas de migración, tendencias demográficas (Bonal y Bellei, 2018). Los factores institucionales, aluden a las regulaciones de los sistemas escolares y en qué medidas sus políticas educativas favorecen la conformación de escuelas heterogéneas. Los factores socioculturales se asocian a las apreciaciones, preferencias y maniobras de las familias al elegir escuela.

Varios autores consideran que el alto nivel de segregación escolar en Chile se relaciona con las políticas neoliberales aplicadas desde la década de los '80 (Bellei, 2015; Bellei et al., 2018; Elacqua, Montt y Santos, 2013; Flores y Carrasco, 2013; Valenzuela, Bellei y de los Ríos, 2014). En dicho periodo los establecimientos educacionales públicos fueron traspasado en términos administrativos a los municipios desde el Ministerio de Educación y se alentó la participación de privados a través del sistema de voucher que sigue a los estudiantes. Con este mecanismo se financian establecimientos públicos y privados subvencionados hasta la actualidad. Las familias son concebidas como protagonistas porque tienen la tarea de escoger la escuela de sus hijos e hijas ${ }^{1}$, generando competencia entre éstas para tener financiamiento. Esta presión, teóricamente, llevaría a las escuelas a entregar un servicio educativo mejor lo que impactaría positivamente en la calidad del sistema (Bellei y Vanni, 2015, en Bellei, 2015). Para ello era fundamental que las familias contaran con información sobre la calidad académica de las escuelas a fin de que pudieran optar por "las mejores" (Hastings y Weinstein, 2008). Con el objeto de favorecer "buenas decisiones" en 1988 fue creado el Sistema de Medición de la Calidad, SIMCE² (Bravo, 2011).

\footnotetext{
${ }^{1}$ En Chile no existen políticas de zonificación que definan la escuela en función de la residencia.

2 Se trata de una prueba estandarizada de carácter censal para ciertos niveles educativos en asignaturas como lenguaje y matemáticas que busca medir los aprendizajes del estudiantado. Sus resultados son públicos a nivel de escuela. A través de esta medición se recaba, además, información diversa relativa a los y las estudiantes (opinión sobre su propio desempeño y distintos aspectos de la vida escolar, entre otros), profesorado (formación, condiciones laborales y valoración sobre funcionamiento del establecimiento) y familias (por ejemplo, composición del hogar, nivel educativo
} 
La literatura especializada muestra que las escuelas pueden competir recurriendo a prácticas como la exclusión de estudiantes con menor rendimiento real o potencial (González González, 2008). Diversos estudios, efectuados tanto en el contexto de una prácticamente inexistente regulación en esta materia (Contreras, Sepúlveda y Bustos, 2010; Madero y Madero, 2012; Parry, 1996) como en el marco de una regulación débil (Carrasco, Gutiérrez y Flores, 2017; Godoy, Salazar y Treviño, 2014), muestran que la selección de estudiantes es frecuente en las escuelas chilenas, aunque en diferente medida dependiendo del tipo de escuela.

Por otra parte, en 1993 se instauró el sistema de financiamiento compartido o copago a través del cual se permite que establecimientos educativos con financiamiento público cobren por sus servicios bajo ciertas condiciones. Pueden hacerlo establecimientos públicos sólo en enseñanza media y con el consentimiento de las familias, mientras que los establecimientos privados subvencionados pueden cobrar en el nivel primario y secundario, sin necesidad de contar con aprobación de las familias. Junto a ello, a medida que el establecimiento cobra más se produce un recorte mayor en el porcentaje de subvención escolar que la escuela percibe. Con todo, el sistema se expandió ampliamente en el sector privado subvencionado (Elacqua, Montt y Santos, 2013). Este sistema se encuentra en funcionamiento hoy en día con normativas sobre el monto que puede cobrar cada establecimiento.

Los movimientos estudiantiles han cuestionado sistemáticamente la mercantilización de la educación. Esto ha incidido en la tramitación de nuevas leyes, destacándose la Ley de Inclusión Escolar (que establece fin al financiamiento compartido, a la selección de estudiantes y al lucro) y la Ley de Nueva Educación Pública (que implica el traspaso de establecimientos desde municipios a Servicios Locales de Educación). Ambas están en proceso de implementación. Para nuestros fines se destaca la Ley de Inclusión en la medida que su objetivo es reformar la estructura del sistema para reducir la segregación escolar y fomentar la integración social. Esta reforma fue percibida como la más significativa del gobierno de Bachelet (2014-2018) por los directores de los establecimientos escolares (Weinstein, Muñoz y Rivero, 2018).

Actualmente el sistema escolar está conformado por tres tipos de escuela:

- Establecimientos municipales (públicos): Se financian por medio de subvención estatal por alumno/a. Históricamente ha existido en ellos menos prácticas de selección. Escolariza al 34\% de los estudiantes (CEM, 2019).

- Establecimientos privados subvencionados: Financiados por subvención escolar y aportes de las familias vía financiamiento compartido. En este sector existen prácticas más extendidas de selección del alumnado. Actualmente, matricula al 54\% de los estudiantes (CEM, 2019).

- Establecimientos privados no subvencionados: Financiados completamente por aranceles que pagan las familias, con prácticas de selección muy extendidas y una matrícula que representa el 9\% (CEM, 2019).

de los padres, nivel de ingresos mensuales, opinión sobre distintos aspectos del establecimiento educativo). Para más detalles se recomienda visitar el sitio web www.agenciadecalidad.cl 
Este trabajo busca analizar discursos de elección de escuela de familias de estos tres tipos de establecimiento. Se concibe la elección de escuela como una práctica sociocultural (Carrasco, Falabella y Mendoza, 2015) que conjuga distintos elementos como fuentes de información y criterios de elección entre otros. El trabajo asumió un enfoque cualitativo. La producción de datos se realizó en dos casos, cada uno de ellos conformados por tres escuelas que reflejan la diversidad existente en Chile. Estudios previos analizan las motivaciones de familias sin considerar la ubicación geográfica de las escuelas. Este trabajo, en cambio, analiza las preferencias de familias que escolarizan a sus hijos en establecimientos de distinto tipo pero que se emplazan a escasos metros entre sí.

\section{Revisión de literatura}

La literatura especializada documenta que la libre elección de escuela influye sobre la segregación escolar porque las familias no se aproximan a este proceso en las mismas condiciones, siendo los sectores más favorecidos quienes se benefician en mayor medida (Ball, 1993, 1995; Ball y Vincent, 1998; Ball, Bowe y Gewirtz, 1996; Broccolichi y Van Zanten, 2000; Murillo et al., 2018; Van Zanten, 2003, 2015). La literatura chilena sobre el tema es amplia, sin embargo, centramos la revisión en estudios cualitativos, ya que tienen el mismo enfoque de este trabajo.

Se ha documentado que las familias de sectores pobres aspiran a escuelas que aseguren la adquisición de aprendizajes, buenos profesores y directivos que mantengan un clima de disciplina (Córdoba, 2014). Los resultados del SIMCE no son percibidos como un reflejo de calidad y éstos no suelen ser utilizados salvo excepciones (Gubbins, 2013; Navarro, 2004; Raczynski et al., 2010). Estas familias buscan ofrecer a sus hijos un espacio en que se reduzcan las desventajas y temores asociados a vivir en barrios estigmatizados (Bellei et al., 2018). Asimismo, esperan que la experiencia escolar resulte placentera gracias al apoyo de docentes, el buen trato y la apertura de la escuela (Gubbins, 2013).

Además, consideran importante que sus hijos tengan el menor contacto posible con estudiantes percibidos como una amenaza o mala influencia por su apariencia, lenguaje, comportamiento, consumo de alcohol o drogas o con familias percibidas como delincuentes (Córdoba, 2014; Hernández y Raczynski, 2015; Navarro, 2004). En este escenario el copago, aun cuando sea bajo, es un mecanismo que permite la exclusión de determinados estudiantes. Cuando las escuelas que se encuentran cercanas a sus domicilios no les ofrecen esta garantía están dispuestos a desplazarse hacia otros sectores (Bellei et al., 2018; Córdoba, 2014;).

Los estudios con familias de nivel socioeconómico medio evidencian que éstas valoran positivamente altos niveles de exigencia (Raczynski et al., 2010), que están más informadas sobre los resultados del SIMCE y consideran éstos en sus decisiones (Rojas, Falabella y Leyton, 2016). Aspiran a que sus hijos lleguen a la universidad y obtengan un estatus social mayor (Bellei et al., 2018; Raczynski et al., 2010; Rojas, Falabella y Leyton, 2016). Un aspecto central es el temor de que sus hijos se mezclen con la "turba", con "flaites", que representan la cultura marginal popular con tendencia a la ostentación (Canales, Bellei y Orellana, 2016). El flaite es, además, la antítesis del habitus profesional que buscan cultivar en sus hijos (Rojas, Falabella y Leyton, 2016). Por ello descartan escuelas ubicadas en barrios percibidos como peligrosos o que atienden estudiantes de mal comportamiento y/o vulnerables (Bellei et al., 2018; Raczynski et al. 2010). 
Como contrapunto, valoran escuelas que escolarizan un alumnado de nivel socioeconómico similar o levemente superior. Suelen preferir escuelas que seleccionan estudiantes y con copago, teniendo una visión negativa de las escuelas gratuitas que no seleccionan (Falabella, Seppänen y Raczynski, 2015; Hernández y Raczynski, 2015; Raczynski et al., 2010; Stillerman, 2016). El trabajo de Bellei y otros (2018) propone una distinción en este segmento entre "nueva clase media" y "clase media tradicional". La primera ha experimentado un proceso de ascensión social reciente que teme perder, $\mathrm{y}$ considera un deber moral entregar a sus hijos la mejor educación a su alcance resguardando permanentemente su distinción de capas sociales más desfavorecidas. La segunda corresponde a familias con mayor capital socioeconómico y cultural, que orientan sus elecciones en función de proyectos educativos amplios y consideran que las escuelas privadas responden a sus aspiraciones.

Madres y padres de niveles socioeconómicos más acomodados escogen casi exclusivamente establecimientos privados no subvencionados. Una opción distinta no aparece en su discurso (Gubbins, 2014) y, aun cuando puedan estar de acuerdo con el fortalecimiento de la educación pública, enviar a los hijos a un establecimiento subvencionado por el Estado es inimaginable (Carrasco, Donoso y Mendoza, 2016). Paradójicamente, algunas de estas familias consideran que su abanico de opciones es limitado porque se ven a sí mismas como parte de una comunidad que envía a sus hijos a un reducido grupo de escuelas. En otros casos, otorgan relevancia al desempeño académico (Bellei et al., 2018), pero el SIMCE no es determinante (Carrasco, Donoso y Mendoza, 2016; Gubbins, 2014).

Valoran el aprendizaje de una segunda lengua, el desarrollo integral, el uso de ciertas metodologías, la promoción de autonomía y el trato personalizado (Carrasco, Donoso y Mendoza, 2016; Gubbins, 2014). Estas familias buscan perpetuar sus privilegios a través de la elección de establecimientos altamente prestigiosos (Gubbins, 2014) a los cuales han asistido ellos, sus familiares y círculo de amigos (Carrasco, Donoso y Mendoza, 2016). Aun cuando pueden observarse diferencias entre estos colegios en cuanto a su orientación religiosa, ideario social o herencia extranjera (Madrid, 2016), son muy homogéneos socioeconómicamente. Para estos padres resulta fundamental que sus hijos se eduquen con otros con quienes compartan estilos de vida, códigos culturales y redes de contacto.

\section{Método}

La investigación se desarrolló por medio de un estudio de casos de tipo descriptivo exploratorio, privilegiando la comprensión profunda de unidades complejas en la perspectiva de analizar un fenómeno en el cual concurren diferentes dimensiones (Della Porta, 2013). Los dos casos presentados en este trabajo están compuestos por pequeños conjuntos de tres escuelas distanciadas entre sí por aproximadamente 200 metros alrededor de las cuales no existen otros establecimientos educativos en un radio de, al menos, 500 metros desde el centroide. Este diseño buscaba analizar el fenómeno de la segregación escolar en una escala mayor a la de estudios previos, efectuados a nivel de municipios o ciudades. Los casos fueron denominados Unidad Geográfica Acotada (UGA) originalmente en el proyecto de investigación en el cual se enmarca este estudio.

Este trabajo da cuenta de los hallazgos efectuados a través de entrevistas en dos UGAs. Cada una de ellas está compuesta por tres establecimientos educativos que representan la 
diversidad de escuelas existentes en el sistema escolar chileno. Las figuras 1 y 2 representan cada caso.

La producción de datos se realizó en 2016 por un periodo de 9 meses, durante los cuales se desarrollaron observaciones no participantes y entrevistas semiestructuradas a familias de niños y niñas de primer ciclo de enseñanza básica $\left(1^{\circ}\right.$ a $4^{\circ}$ año), que enviaban a sus hijos a alguna de las 6 escuelas que componen los dos casos de estudio. Se realizó un total de 54 entrevistas, 27 en cada UGA, 9 en cada centro educativo. Los tópicos abordados aludían a las fuentes de información usadas para decantarse por una escuela, los criterios prácticos puestos en juego (como costo y cercanía) y los criterios de calidad educativa. Las entrevistas fueron íntegramente transcritas, desarrollándose un análisis de contenido en base a ellas.

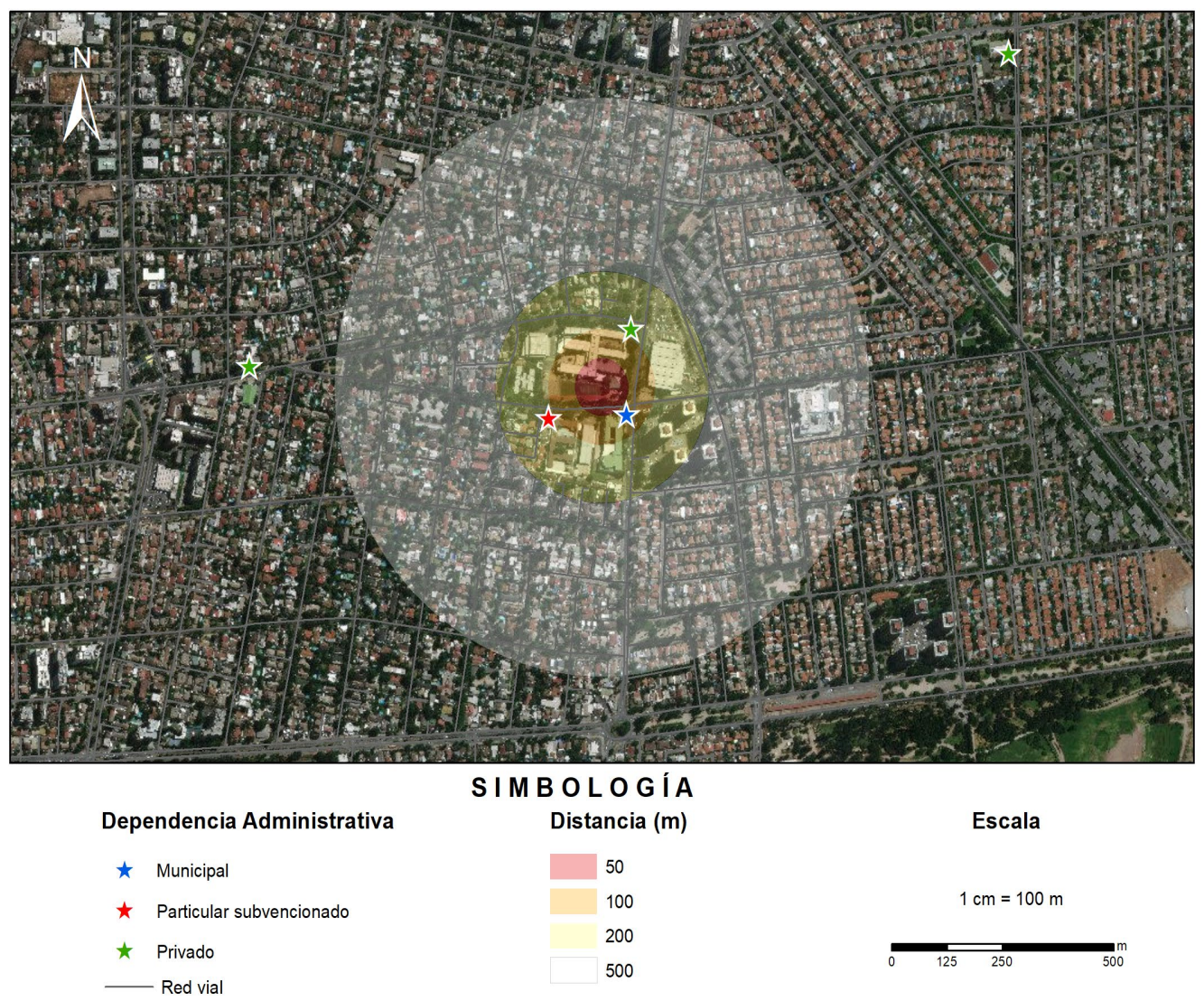

Figura 1. Mapa Unidad Geográfica Acotada UGA 1

Fuente: Elaboración propia.

\section{Caracterización de los casos}

Los casos resultan de gran interés porque encontrándose las escuelas insertas dentro de un mismo barrio y separadas entre sí por escasos metros reflejan las diferencias que existen entre las escuelas del sistema escolar chileno.

La UGA 1 se emplaza en un municipio del sector oriente de la capital que alberga preferentemente familias de nivel socioeconómico medio-alto y alto. La UGA 2 se ubica en una comuna de Santiago sur. Su población es más heterogénea en términos socioeconómicos. 


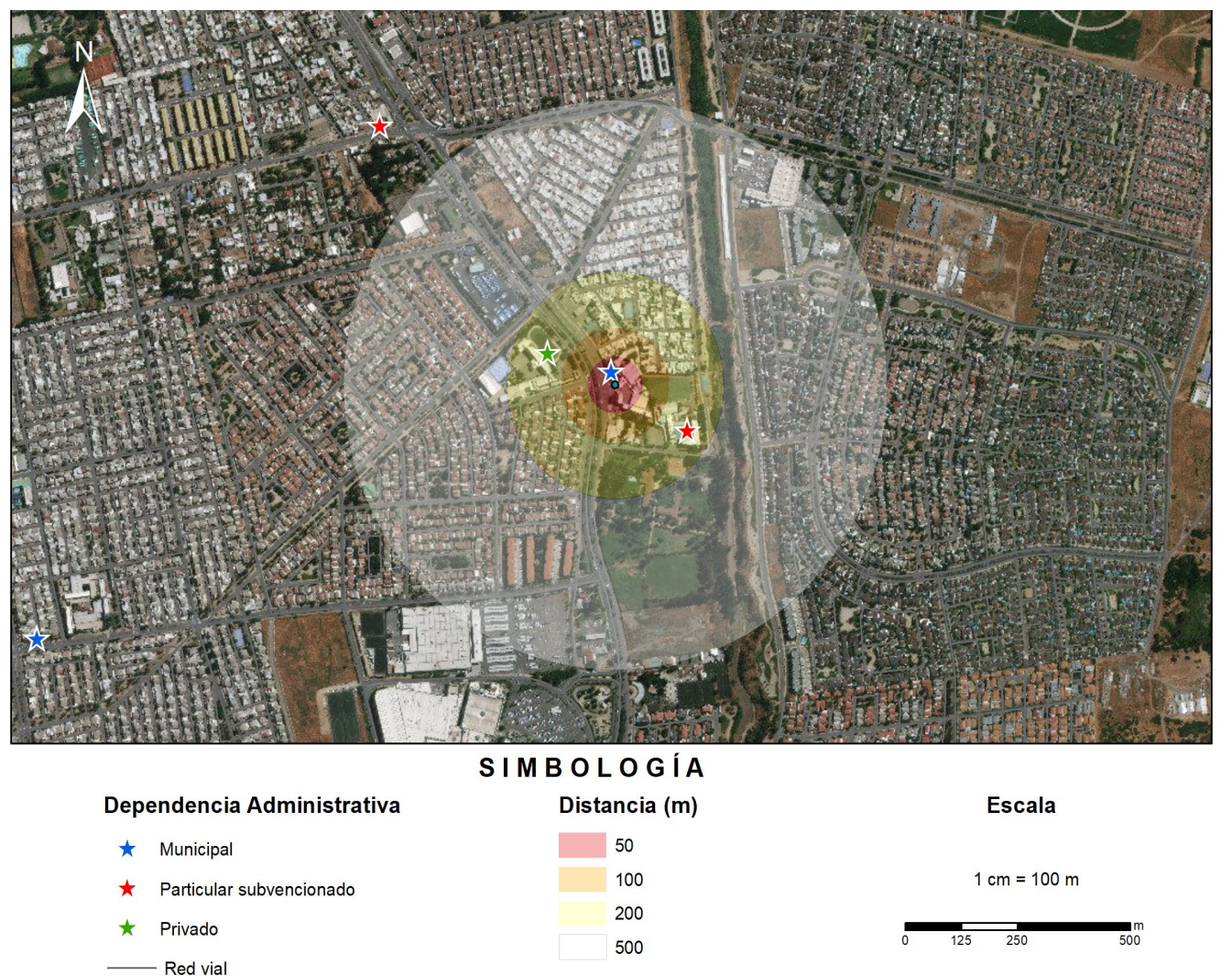

Figura 2. Mapa Unidad Geográfica Acotada UGA 2

Fuente: Elaboración propia.

El cuadro 1 informa sobre los costos para las familias por matrícula, aranceles y otros. Como puede apreciarse, las escuelas municipales son gratuitas. Ambas, así como la escuela privada subvencionada de la UGA 2, están adscritas al Sistema de Subvención Escolar Preferencial, en adelante "SEP", que implica que los estudiantes identificados como prioritarios no requieren pagar si la escuela cobra por financiamiento compartido y que la subvención otorgada por ellos es mayor. En las demás escuelas privadas, en cambio, se debe pagar por concepto de matrícula y financiamiento compartido o aranceles. Existen considerables diferencias en los montos solicitados por estas escuelas, sobre todo en la UGA 1 donde el arancel de la privada no subvencionada es 8 veces superior al de la privada subvencionada. En el caso de la UGA 2 la escuela privada no subvencionada cobra el triple de la privada subvencionada.

La composición socioeconómica del alumnado de las escuelas de las mismas UGAs es notablemente distinto. El cuadro 2 ofrece datos provenientes de SIMCE 2015 para $4^{\circ}$ básico. Como puede apreciarse los establecimientos municipales se caracterizan por escolarizar en mayor medida a estudiantes cuyas madres y padres tienen menores niveles educativos e ingresos mensuales, mientras las familias de escuelas privadas no subvencionadas están en la situación opuesta, ocupando las familias de escuelas privadas subvencionadas una posición intermedia. Esta tendencia se confirma analizando otros datos de composición socioeconómica del estudiantado. El Índice de Vulnerabilidad 
Escolar (en adelante "IVE" 3 ) de 2015 para enseñanza básica, es mayor en las escuelas municipales que en las privadas subvencionadas: $43,8 \%$ y $35,5 \%$ en la UGA 1 y $79,7 \%$ y $60,1 \%$ en la UGA 2.

Cuadro 1. Costes asumidos por familias en cada escuela, UGAs 1 y 2 (2016, en pesos chilenos)

\begin{tabular}{|c|c|c|c|}
\hline $\begin{array}{c}\text { Caso / } \\
\text { Costos }\end{array}$ & MUNICIPAL & PRIVADA SUBVENCIONADA & PRIVADA NO SUBVENCIONADA \\
\hline UGA 1 & $\begin{array}{l}\text { Matrícula }=0 \\
\text { Arancel }=0 \\
\text { Adscrita a SEP }\end{array}$ & $\begin{array}{l}\text { Matrícula }=\$ 10.000 \\
\text { Arancel }=\$ 25.000-\$ 40.000 \\
\text { No adscrita a SEP }\end{array}$ & $\begin{array}{l}\text { Postulación }=\$ 35.000 \\
\text { Cuota de incorpor. }=\$ 1.300 .000 \\
\text { Matrícula }=\$ 400.000 \\
\text { Arancel }=\$ 340.000\end{array}$ \\
\hline UGA 2 & $\begin{array}{l}\text { Matrícula }=0 \\
\text { Arancel }=0 \\
\text { Adscrita a SEP }\end{array}$ & $\begin{array}{l}\text { Matrícula }=\$ 25.000 \\
\text { Arancel }=\$ 50.000 \\
\text { Adscrita a SEP }\end{array}$ & $\begin{array}{l}\text { Matrícula }=\$ 150.000 \\
\text { Arancel }=\$ 150.000\end{array}$ \\
\hline
\end{tabular}

Fuente: Elaboración propia en base a información recabada en trabajo de campo.

Cuadro 2. Composición socioeconómica alumnado, UGA 1 y UGA 2

\begin{tabular}{|c|c|c|c|c|c|c|}
\hline & \multicolumn{3}{|c|}{ UGA 1} & \multicolumn{3}{|c|}{ UGA 2} \\
\hline & $\mathbf{M}$ & PS & PNS & $\mathbf{M}$ & PS & PNS \\
\hline $\begin{array}{l}\text { Porcentaje de madres que no completó } \\
\text { enseñanza obligatoria }\end{array}$ & $22,6 \%$ & $13,8 \%$ & $\mathrm{O} \%$ & $50 \%$ & $15 \%$ & $1,4 \%$ \\
\hline $\begin{array}{l}\text { Porcentaje de madres que completó } \\
\text { enseñanza superior }\end{array}$ & $29,2 \%$ & $52,3 \%$ & $93,8 \%$ & $5 \%$ & $37,5 \%$ & $74,6 \%$ \\
\hline $\begin{array}{l}\text { Porcentaje de padres que no completó } \\
\text { enseñanza obligatoria }\end{array}$ & $30,2 \%$ & $15,4 \%$ & $1,3 \%$ & $50 \%$ & $20 \%$ & $\mathrm{O} \%$ \\
\hline $\begin{array}{l}\text { Porcentaje de padres que completó } \\
\text { enseñanza superior }\end{array}$ & $24,5 \%$ & $44,6 \%$ & $90 \%$ & $7,5 \%$ & $22,5 \%$ & $73,2 \%$ \\
\hline Familias que viven con menos de $\$ 300.000$ & $26,4 \%$ & $15,4 \%$ & $0 \%$ & $42,5 \%$ & $25 \%$ & $\mathrm{O} \%$ \\
\hline Familias que viven con más de $\$ 1.400 .000$ & $9,4 \%$ & $23,1 \%$ & $88,8 \%$ & $0 \%$ & $5 \%$ & $54,9 \%$ \\
\hline
\end{tabular}

Nota: M: Municipal - PS: Privado Subvencionado - PNS: Privado No Subvencionado.

Fuente: Elaboración propia sobre BBDD SIMCE, $4^{\circ}$ básico, 2015.

\section{Resultados}

\subsection{Fuentes de información y proceso de admision}

En relación con las fuentes de información, las entrevistadas ${ }^{4}$ de la UGA 1 (en todas las escuelas) consideraron recomendaciones de conocidos, familiares o amigos, implicando relaciones e interacción social. Esta tendencia se constata entre las familias de la escuela

\footnotetext{
${ }^{3}$ Este índice busca caracterizar a los y las estudiantes en cuanto a sus necesidades respecto, por ejemplo, a estado de salud o condición de pobreza. Se utiliza para la focalización de políticas gubernamentales y entrega de servicios o beneficios. Es calculado para los establecimientos educativos sostenidos con fondos públicos en base a información aportada por las familias. Su valor oscila entre $0 \%$ y 100\% y su elaboración está a cargo de la Junta Nacional de Auxilio Escolar y Becas JUNAEB. Para más detalles se recomienda visitar el sitio web www.junaeb.cl.

${ }^{4}$ En la presentación de resultados se utiliza preferencialmente el género femenino porque de un total de 54 entrevistas, 45 fueron efectuadas con madres.
} 
municipal (donde gran parte de las entrevistadas eran exalumnas de la escuela) y la privada subvencionada de la UGA 2 :

Bueno yo estudie en el [escuela privada subvencionada] cuando era chica [...] entonces ya lo conocía, por una cosa más de cariño. (Madre, privada subvencionada, UGA 1)

Yo lo conozco desde cuando yo estudiaba. Cuando entró mi hija mayor, estaba la mayoría de los profesores [con los] que yo estudiaba, así que más por eso, por la confianza. (Madre, municipal, UGA 2)

Quienes escolarizan a sus hijos en escuelas privadas subvencionadas (en ambas UGAs) y aquellas que optaron por la escuela privada no subvencionada de la UGA 2, este tipo de conocimiento se complementa con la fuente de información "fría" por excelencia: pruebas estandarizadas como SIMCE. Esta información no es usada por las madres de la escuela privada no subvencionada de la UGA 1.

...por internet, en realidad mi esposo es el que buscó por internet todos los rankings, PSU, SIMCE, siempre en realidad está viendo eso, siempre viendo nivel, en qué posición. (Madre, privada subvencionada, UGA 1)

La PSU, el SIMCE, me acuerdo que me decía, 'mira el mejor colegio aquí es éste que está acá', o no sé cuál, después siguió, y así... eso fue también una forma de conectarnos o de saber del colegio, en internet, las noticias. (Madre, privada no subvencionada, UGA 2)

Las entrevistadas en ambas UGAs informaron que los procesos de admisión fueron simples y poco engorrosos. Es más, la escuela privada no subvencionada de la UGA 1 se diferencia de otros establecimientos de élite que utilizan procedimientos largos y estresantes. La única excepción es la escuela privada no subvencionada de la UGA 2 , en que las familias presentaron diversos documentos (como certificados de notas y comprobantes de ingresos) además de un examen de admisión.

\subsection{Criterios de elección centrados en aspectos prácticos}

Las madres de escuelas municipales de ambas UGAs, enfatizan en que la gratuidad fue determinante en su decisión. Se desprende de sus relatos la creencia de que la calidad se asocia directamente con el costo, pero dadas sus restricciones económicas no tienen más alternativa que una escuela gratuita.

\section{El [colegio privado no subvencionado] es más caro que tener dos hijos en la universidad. To pienso que la educación ahí en el [colegio privado no subvencionado] es súper buena, porque con plata todo es espectacular, si yo tuviera el dinero lo pongo en el [colegio privado no subvencionado], pero como no las tengo, lo pongo en el [colegio municipal]. (Madre, municipal, UGA 1)}

Las madres que escolarizan a sus hijos en escuelas privadas subvencionadas señalan no poder pagar una escuela privada sin subvención que, simbólicamente, representa para ellos la "mejor" educación. Aparece entonces la escuela privada subvencionada como una alternativa para quienes evitan escolarizar a sus hijos en establecimientos municipales.

\footnotetext{
... a mí me complica lo caro de los colegios particulares, y que a veces la educación no es la más recomendada, y... la calidad de los colegios municipales, entonces a veces uno quisiera optar a algo más económico, pero sabe que a veces puede ir un poco en contra de la calidad, por lo tanto, hay que tratar de encontrar como el equilibrio, pero sí que hay mucha diferencia. (Madre, privada subvencionada, UGA 1)
}

Las familias que optan por escuelas privadas no subvencionadas también asumen que la escuela escogida representa "la mejor que pueden pagar". Generalmente estas familias 
manifiestan que pueden sobrellevar los costos de la escolarización sin mayores dificultades. Sin embargo, algunas de la UGA 1, reconocen endeudarse o renunciar a determinadas comodidades.

Tenemos deuda, con el banco y con parientes, podríamos no tener deuda o podríamos tener vacaciones más ricas, cosas así. Nosotros privilegiamos la educación, la salud, que los niños reciban... o sea hacemos un montón de sacrificios. (Madre, privada no subvencionada, UGA 1)

Se constata de esta forma, una naturalización de la existencia de espacios escolares segregados en términos económicos: en la medida que las escuelas pueden o no cobrar y, si lo hacen, existen diferentes rangos de precios, cada familia se posiciona en aquel "nicho de mercado" que le corresponde según sus recursos económicos.

Los colegios se van formando de acuerdo al estatus o al nivel socioeconómico que tienen las familias, uno va viendo, ya, yo puedo pagar este colegio. (Madre, privada no subvencionada, UGA 2 )

Por otra parte, la cercanía entre casa y escuela es un criterio que las madres de escuelas municipales y privadas subvencionadas ponen en juego. Siempre que sea posible, optan por una escuela cercana para facilitar la vida cotidiana y reducir los gastos en transporte. Sin embargo, dadas sus restricciones económicas, no les es posible asumir a la vez gastos de escolarización y transporte, debiendo "renunciar" siempre a algo (cercanía o escuela con copago, por ejemplo).

\section{El [colegio privado no subvencionado] está súper lejos de lo que nosotros podemos pagar. Así que ésta fue la única opción por el tema de la cercanía, porque un colegio más lejos tiene el tema del furgón. (Madre, privada subvencionada, UGA 2)}

La relevancia de la cercanía es menos preponderante para padres de escuelas privadas no subvencionadas que, habitualmente, pueden resolver el tema del traslado más cómodamente a través del uso de vehículo particular o transporte escolar.

Cercanía también, pero minoritaria, hubiese estado ahí un par de kilómetros más lejos no hubiera sido problema para no haber ido ahí. (Madre, privada no subvencionada, UGA 2)

\subsection{Criterios de elección centrados en calidad educativa}

Todas las familias ponen en juego criterios de calidad educativa. Sin embargo, éste es un concepto que cambia entre distintos grupos. Nuestros hallazgos indican que existen similitudes en la noción de calidad que construyen las madres en escuelas municipales y en escuelas privadas subvencionadas en ambas UGAs.

Las madres de escuelas municipales valoran la adquisición de aprendizajes, dispositivos de apoyo para niños y niñas con necesidades educativas especiales y la existencia de relaciones cercanas entre los miembros de la comunidad educativa. Las entrevistadas de la escuela municipal de la UGA 1 destacan que dependa administrativamente de una municipalidad con altos recursos económicos, porque eso implica ciertos apoyos (red multidisciplinaria de profesionales, implementación de programas, provisión de recursos tecnológicos, entre otros) con los que no cuentan escuelas municipales de otras comunas ni escuelas privadas subvencionadas del mismo municipio.

Yo sé que esta comuna igual es una comuna bien preocupada por los colegios, o sea estructuralmente hablando todos súper buenos, tiene actividades extraprogramáticas, nada que envidiarle a un particular. (Madre, municipal, UGA 1) 
Las familias de la escuela municipal de la UGA 2 destacan fervientemente su "calidad humana" aludiendo con ello a aspectos como el cuidado hacia los estudiantes y las relaciones de colaboración que se dan al interior de la comunidad escolar. Este aspecto es tan relevante que, en algunos casos, pueden estar dispuestos a renunciar a una escuela más “exitosa” en términos académicos.

Con mi hijo mayor tuve la experiencia de tenerlo en el colegio de atrás, el particular subvencionado, y ahi es mucho mejor la educación en cuanto a materia, van más avanzados que acá en el liceo, pero en cuanto a calidad humana, es el punto que yo elegí la escuela municipal. (Madre, municipal, UGA 2)

Las madres de escuelas privadas subvencionadas, por su parte, ponen el acento prioritariamente en el aprendizaje y, en este escenario, sí puede ocupar un lugar el rendimiento en pruebas estandarizadas. Estas familias valoran positivamente avances en lectoescritura y matemáticas ajustados al currículum nacional, talleres y actividades extraprogramáticas, logros académicos (individuales y colectivos) y la formación valórica que pueda brindar la escuela.

Hay mucha diferencia, de hecho, por eso yo al (niño) lo tengo arriba (comuna del caso de estudio), porque, aunque sea un colegio subvencionado, igual es diferente, el sistema de estudio, el nivel de exigencia. (Madre, privada subvencionada, UGA 1)

El colegio es casi la segunda casa (...) el colegio es súper importante porque en el fondo le está dando herramientas para que ese niño, independiente de lo que pase en su casa siga su desarrollo. (Madre, privada subvencionada, UGA 2)

Sin embargo, algunas se muestran críticas respecto del alto nivel de exigencia académica al que están sometidos los niños de la escuela privada subvencionada de la UGA 1: los contenidos serían abordados en forma rápida, se dan demasiadas tareas, la metodología de aprendizaje se basa en la memorización de contenidos sin incluir innovaciones.

A diferencia de lo que sucede entre entrevistadas de escuelas municipales y privadas subvencionadas, los discursos de las madres de escuelas privadas no subvencionadas de la UGA 1 y 2 difieren considerablemente. Es importante destacar que se trata de familias que, aun cuando hayan elegido establecimientos privados no subvencionados, tienen perfiles socioeconómicos y culturales diferentes. Como se puede apreciar en el Cuadro 2 , las familias de la escuela privada no subvencionada de la UGA 1 aventajan a las de la UGA 2 en nivel educativo e ingresos económicos. Asimismo, existen notables diferencias en los territorios en que se insertan los establecimientos: uno se emplaza en un municipio de élite, mientras el otro se ubica en una comuna heterogénea que ha albergado en los últimos años a clase media emergente a través de la construcción de condominios orientados a este segmento.

El criterio de calidad educativa que buscan los apoderados de la escuela privada no subvencionada de la UGA 1 se relaciona con el proyecto educativo del establecimiento. Éste se caracteriza por: sello de inclusión, énfasis en la formación valórica, metodología de aprendizaje diferente a la tradicional, oferta de actividades extraprogramáticas, infraestructura de gran nivel. Esto lo distinguiría de otros colegios enfocados en el rendimiento académico y la competitividad.

La opción por este establecimiento no está libre de contradicciones. Las entrevistadas son conscientes de las "desventajas" que puede tener este establecimiento en comparación a otros establecimientos de élite, en cuanto a énfasis en deportes, aprendizaje de segunda 
lengua o rendimiento en pruebas estandarizadas. Sin embargo, las características de su proyecto educativo compensan estas debilidades.

Empezó a prevalecer el tema valórico, de la inclusión, que pudieran conocer a otro tipo de gente, sabiendo que lo otro, que el deporte, que el inglés, podía ser subsanado por otra parte... (Madre, privada no subvencionada, UGA 1)

El concepto de calidad educativa para las madres de la escuela privada no subvencionada de la UGA 2 es distinta. Ésta se asocia fuertemente con los costos y, por tanto, consideran que mientras más paguen mayores posibilidades tienen de acceder a una mejor educación, que se traducirá en altos puntajes en pruebas estandarizadas y en la continuidad de estudios terciarios en instituciones de prestigio. En un segundo plano se valoran otros aspectos, como altos niveles de disciplina, la práctica de actividades deportivas o artísticas y la formación valórica.

Todos me comentaban que el otro colegio particular pagado de la comuna no era tan bueno. Que era como... bueno, en los puntajes SIMCE se veía más abajo que nuestro colegio, eso también le da un punto. (Madre, privada no subvencionada, UGA 2)

\title{
4.4. Criterio de elección centrados en la composición social y cultural
}

El último conjunto de criterios considerados por las familias de los tres tipos de escuela es la composición social y cultural del estudiantado: se evade cierto perfil de compañeros y se busca un grupo similar al propio en términos socioeconómicos y culturales.

La tendencia general es la evitación del contacto con niños y familias de nivel socioeconómico más bajo, de comportamiento agresivo, desafiante con la autoridad, que usan un lenguaje vulgar, que pueden tener alguna cercanía con el mundo de la delincuencia y/o drogas. Todas estas características son condensadas en la figura del "flaite", de quien rehúyen los padres de los tres tipos de escuela, salvo dos excepciones.

Los padres de la escuela municipal de la UGA 1 están conscientes de que sus características institucionales facilitan el ingreso de niños y niñas percibidos como mala influencia o amenaza. Al no tener alternativas confían en los recursos de sus hijos, en su propia influencia, o bien, reformulan esta desventaja valorando que se eduquen en un espacio escolar heterogéneo.

\begin{abstract}
...lo que pasa en [comuna] igual hay harta droga, hay harta familia que son traficantes y muchos de ellos van al colegio o de papás que han estado en la cárcel, que son delincuentes [...] entonces pasa eso, yo trato de luchar contra eso, y digo pucha si hay de todo no más, que le vamos a hacer [...] ella se va a encontrar y mezclar con cualquier tipo de gente después, no hay que ponerla en una burbuja. (Madre, municipal, UGA 1)
\end{abstract}

Este temor se ve en parte contrarrestado por el hecho de que la escuela se ubica en una comuna de alto nivel socioeconómico y alejada del sector más desfavorecido que existe dentro del municipio. Así, la ubicación geográfica de la escuela representa una ventaja comparativa. La misma idea es señalada por padres de la escuela privada subvencionada vecina.

... de hecho por eso lo metí en un subvencionado arriba, y no en un subvencionado acá en la comuna (sector poniente de Santiago), porque un subvencionado acá en la comuna es lo mismo... es lo mismo que un municipal, no hay mucha diferencia. (Madre, privada subvencionada, UGA 1)

El temor al "contagio" se ve exacerbado en padres de escuelas privadas subvencionadas (de ambas UGAs) y no subvencionada de la UGA 2, quienes consideran que las escuelas 
municipales vecinas escolarizan, justamente, niños que se busca evitar, descartándolas para sus hijos. Para estos padres las barreras de entrada que pueden poner sus escuelas, como el copago y procesos de selección son positivamente valoradas porque jugarían un rol protector al disminuir la posibilidad de ingreso de estudiantes "indeseados".

... pasa que ahí en [colegio municipal vecino] vienen chicos de lugares, de [la comuna], donde hay mucha delincuencia, droga, entonces son otro tipo de alumnos. Entonces, yo creo que ahi uno empieza a buscar donde su hijo va a estar mejor y no se va a contagiar de todas estas cosas complejas de tratar... Entonces, por lo que uno escucha, es más complicado el tipo de alumno del [establecimiento municipal]. (Madre, privada subvencionada, UGA 1)

Mucho flaite, muchos niños que a lo mejor su forma de presentarse, de ir al colegio, no es la adecuada y es una forma de discriminar, porque es una forma de discriminar. Lamentablemente, con recursos tú puedes ir apartando un poco de esa situación a tus hijos. No es que esté en contra de ellos, pero es mi temor y mi temor radica mucho en que, si llevo a los niños a un colegio municipal, el gran porcentaje de estos niños puedan traer a mis hijos a un mundo distinto al cual yo no quiero que ellos lleguen; drogadicción, alcoholismo, maltrato. (Madre, privada no subvencionada, UGA 2)

Las madres de la escuela privada no subvencionada de la UGA 2, además de intentar evitar a los "flaite", buscan un ambiente socioeconómico y cultural homogéneo en que sus hijos se relacionen con niños y familias muy similares a ellos.

Por las personas que iban al colegio, porque nosotros mirábamos alrededor de lo que teníamos y no queríamos que se relacionaran, de repente, con personas, para decirlo de manera franca, como bien sensata, con personas que tuvieran, a lo mejor, un nivel de estudio distinto al que tenemos nosotros. Acá uno sabe que la mayoría de los papás son profesionales, entonces vamos buscando eso. (Madre, privada no subvencionada, UGA 2)

La preocupación por las características socioeconómicas y culturales de los compañeros de los hijos no se observa en dos de las escuelas estudiadas que, de alguna manera, representan casos extremos. Las familias de la escuela municipal de la UGA 2 saben que son percibidas por otros como "flaites". Frente a esta denostación actúa como protector el fuerte sentido de pertenencia a su comunidad educativa.

Meten a todos en el mismo saco entonces creen que todos somos iguales. Nos comparan con la gente de allá, [de una población del sector]. (Madre, Municipal, UGA 2)

Por su parte, las familias de la escuela privada no subvencionada de la UGA 1 no aluden al temor al "contagio" porque no se exponen a éste: los costos económicos de esta escuela la restringen a familias de nivel socioeconómico alto.

\section{Discusión y conclusiones}

Nuestros hallazgos coinciden con la literatura previa en varios sentidos. Se pone en evidencia, nuevamente, que la "libre" elección de escuela está condicionada por los recursos económicos que definen el rango de opciones en el "mercado escolar".

La cercanía es valorada por familias de distintos tipos de escuela, no obstante, es una prioridad a la que están dispuestos a renunciar cuando las escuelas cercanas no cumplen con su criterio de calidad. Las fuentes de información que predominan son las recomendaciones y experiencias de familiares y amigos. El SIMCE tiene un lugar secundario, excepto para algunas familias que optan por escuelas privadas (con o sin 
subvención). La noción de calidad que construyen las familias es compleja y diversa pudiendo abarcar diferentes elementos de acuerdo con su nivel socioeconómico.

Los discursos de las madres respecto a cómo eligen escuela permiten observar la construcción y definición del "nosotros" y "los otros" en un sentido colectivo. Con esas definiciones las familias determinan con quienes desean que sus hijos compartan escuela y quiénes son "indeseados". En línea con ello, algunas familias de mayores recursos socioeconómicos escogen escuela con el objetivo explícito o implícito de distinguirse o protegerse de otros, buscando espacios educativos de homogeneidad socioeconómica y cultural.

Todo lo anterior concuerda con el cuerpo de conocimiento acumulado en torno al proceso de elección de escuela (Bellei et al., 2018, Córdoba, 2014; Gubbins, 2013; Raczynski et al., 2010; Rojas, Falabella y Leyton, 2016; entre otros) y evidencia que las valoraciones y comportamientos de las familias inciden sobre la segregación socioeconómica del sistema escolar. Asimismo, este trabajo contribuye a comprender cuáles son los significados de calidad educativa que las familias ponen en juego al momento de escoger escuela (Román y Murillo, 2014).

El diseño metodológico del estudio permite hacer aportaciones novedosas. En primer término, las UGAs están conformadas por escuelas muy desiguales en la composición socioeconómica de su estudiantado, aun cuando se trata de establecimientos vecinos. Se demuestra que la segregación socioeconómica escolar puede ocurrir a una escala mayor de la que se ha utilizado en estudios previos en Chile, habitualmente de comunas. Sería necesario entonces tener en cuenta que los límites administrativos pueden no representar territorios homogéneos (Ruiz Tagle y López, 2014) y esta complejidad territorial debería ser considerada en estudios sobre segregación escolar. En segundo término, la cercanía física no implica cercanía social (Ruiz Tagle, 2016); diferentes grupos pueden compartir un mismo territorio y desplegarse en éste como si vivieran en mundos paralelos. Este trabajo refleja aquello, porque los discursos de elección de escuela de las familias se encuentran claramente definidos por su estatus socioeconómico, independientemente de la distancia física entre escuelas.

Por otra parte, refrendamos que la ubicación geográfica de los establecimientos también es tenida en cuenta, no sólo desde la perspectiva de la cercanía con la casa o trabajo, sino de las características del barrio. Raczynski y otros (2010), documentan que madres y padres de nivel socioeconómico medio descartan escuelas ubicadas en barrios percibidos como peligrosos, porque a ellas llegarían más fácilmente los estudiantes que quieren evitar. Asimismo, este trabajo muestra que la ubicación en una comuna privilegiada y en un sector específico de ésta, vuelve más atractiva una escuela porque disminuiría esta posibilidad.

En la misma línea, es necesario recalcar que la producción y reproducción de la segregación escolar está relacionada con la configuración socioespacial de la ciudad y el fenómeno de la segregación residencial. Ésta es particularmente intensa para grupos de altos ingresos en Santiago (Agostini et al., 2016) que tienden a la autosegregación residencial (Vignoli y Rowe, 2019). Esto evidencia la necesidad de analizar la segregación escolar en relación con la residencial (Stillerman, 2016) y de investigar a grupos sociales abiertos a la integración territorial y escolar (Urrutia et al., 2017). 
Finalmente, este trabajo aporta información sobre la diversidad en las lógicas de elección de familias que optan por escuelas privadas no subvencionadas. Por una parte (UGA 2) encontramos familias que se informan preferentemente por fuentes oficiales y estandarizadas sobre la "calidad académica", evalúan las "ofertas" del "mercado escolar" según precio/calidad y están preocupadas del tipo de compañero de sus hijos. Se trata de familias que coinciden con el perfil "nueva clase media" propuesto por Bellei y otros (2018), siendo el único grupo que se acerca en mayor medida al comportamiento ideal de la teoría de la libre elección de escuela. En la UGA 1 las familias representan un perfil inusual, porque privilegian un proyecto educativo con sello de inclusión, metodologías de aprendizaje innovadoras y formación valórica. Su perfil es más cercano a la "clase media tradicional" o "clase alta" (Bellei et al., 2018).

Identificamos dos debilidades en este trabajo. La primera es que se trata de un estudio basado únicamente en dos casos. La segunda es que por motivos de espacio no se ahonda en resultados que permitirían complementar algunas de las conclusiones presentadas, como los obtenidos a través de las observaciones en los barrios.

El estudio de la segregación escolar en Chile tiene diversas aristas que será necesario abordar en futuras investigaciones. En primer término, es imprescindible analizar el efecto que tendrá el nuevo Sistema de Admisión Escolar SAE implementado en el marco de la aplicación de la Ley de Inclusión. A través de éste se pone fin a los procesos de selección de estudiantes por parte de escuelas sostenidas con fondos públicos, lo que teóricamente implica mayores posibilidades de elección para las familias. Sin embargo, los estudios que analizan sus opciones en este nuevo contexto muestran que las pautas de elección (especialmente de los grupos más desaventajados) gozan de bastante estabilidad (Carrasco y Honey, 2019; Sillard, Garay y Troncoso, 2018). Esto se explica en parte porque debido a la implementación gradual de la medida del fin al copago, aún hay establecimientos con este mecanismo de financiamiento.

Junto a ello, la incorporación creciente de estudiantes migrantes en el sistema escolar chileno sugiere la aparición de nuevas formas de segregación escolar: este alumnado está siendo atendido preferentemente por establecimientos públicos (CEM, 2019) y, además, dentro de este sector su distribución no se produce de forma homogénea siendo algunas escuelas las que tienden a escolarizarlos en mayor medida (Castillo, Santa Cruz y Vega, 2018).

A la luz de estas consideraciones la construcción de un sistema escolar más integrado en términos académicos y socioeconómicos continuará siendo uno de los desafíos más relevantes del sistema escolar chileno para los años venideros.

\section{Agradecimientos}

Este trabajo se enmarca en el Proyecto FONDECYT de Iniciación a la Investigación $\mathrm{N}^{\circ} 11130149$. Se agradece el apoyo del Programa.

Se utilizó como fuente de información las bases de datos de la Agencia de Calidad de la Educación. Los autores agradecen a la Agencia de Calidad de la Educación el acceso a la información. Todos los resultados del estudio son de responsabilidad de los autores y en nada comprometen a dicha institución. 
Se agradecen los comentarios y sugerencias de los revisores de la primera versión de este trabajo, así como la colaboración de Manuel Vallejos, autor de los mapas.

\section{Referencias}

Agencia de Calidad de la Educación. (2015). Base de datos SIMCE $4^{\circ}$ básico 2015. Ministerio de Educación.

Agostini, C., Hojman, D., Román, A. y Valenzuela, L. (2016). Segregación residencial de ingresos en el Gran Santiago, 1992-2002: Una estimación robusta. EURE, 42(127), 159-184. https://doi.org/10.4067/So250-71612016000300007

Ball, S. (1993). Education markets, choice and social class: The market as a class strategy in the UK and the USA. British Journal of Sociology of Education, 14(1), 3-19. https://doi.org/10.1080/0142569930140101

Ball, S. (1995). Circuits of schooling: A sociological exploration of parental choice of school in social class context. Sociological Review, 43(1), 52-78. https://doi.org/10.1111/j.1467-954X.1995.tbo2478.x

Ball, S. y Vincent, C. (1998). I heard it on the grapevine: "Hot” knowledge and school choice. British Journal of Sociology of Education, 19(3), 377-399. https://doi.org/10.1080/0142569980190307

Ball, S. J., Bowe, R. y Gewirtz, S. (1996). School choice, social class and distinction: The realization of social advantage in education. Journal of Education Policy, 11(1), 89-112. https://doi.org/10.1080/0268093960110105

Bellei, C. (2013). El estudio de la segregación socioeconómica y académica de la educación chilena. Estudios Pedagógicos, 39(1), 325-345. https://doi.org/10.4067/S0718-07052013000100019

Bellei, C. (2015). El gran experimento. Mercado y privatización de la educación chilena. LOM. https://doi.org/10.18800/educacion.201802.011

Bellei, C. y Vanni, X. (2015). Evolución de las políticas educacionales en Chile: 1980-2014. En Bellei, C., El gran experimento: Mercado y privatización de la educación chilena (pp. 23-45). LOM.

Bellei, C., Contreras, M., Canales, M. y Orellana, V. (2018). The production of socio-economic segregation in chilean education. School choice, social class and market dynamics. En X. Bonal y C. Bellei, Understanding school segregation. Patterns, causes and consequences of spatial inequalities in education (pp. 221-240). Bloomsbury.

https://doi.org/ 10.5040/9781350033542.ch-011

Bonal, X. y Bellei, C. (2018). The renaissance of school segregation in a context of globalization. En X. Bonal y C. Bellei (Eds.), Understanding school segregation. Patterns, causes and consequences of spatial inequalities in education (pp. 1-26). Bloomsbury. https://doi.org/10.5040/9781350033542.ch-001

Bravo, J. (2011). SIMCE: Pasado, presente y futuro del sistema nacional de evaluación. Estudios Públicos, 123, 189-211.

Broccolichi, S. y Van Zanten A. (2000). School competition and pupil flight in the urban periphery. Journal Education Policy, 15(1), 51-60. https://doi.org/10.1080/026809300286015

Canales, M., Bellei, C. y Orellana, V. (2016), ¿Por qué elegir una escuela privada subvencionada? Sectores medios emergentes y elección de escuela en un sistema de mercado. Estudios Pedagógicos, 42(3), 89-109. https://doi.org/10.4067/S0718-07052016000400005 
Carrasco, A. y Honey, N. (2019). Nuevo sistema de admisión escolar y su capacidad de atenuar la desigualdad de acceso a colegios de calidad: Al inicio de un largo camino. Centro Justicia Educacional.

Carrasco, A., Donoso, A. y Mendoza, M. (2016). La dimensión ético-política de la elección de escuela: dilemas en familias chilenas de elite. En J. Corvalán, A. Carrasco y J. E. García Huidobro (Eds.), Mercado escolar y oportunidad educacional. Libertad, diversidad y desigualdad (pp. 301-335). CEPPE, Ediciones UC. https://doi.org/10.1163/9789463002622_001

Carrasco, A., Falabella, A. y Mendoza, M. (2015). School choice in Chile as a sociocultural practice: An ethnographic inquiry. En P. Seppänen, A. Carrasco, M. Kalalahti, R. Rinne y H. Simola (Eds.), Contrasting dynamics in education politics of extremes: School choice in Finland and Chile (pp. 245-266). Sense Publishers. https://doi.org/10.1163/9789463002622_012

Carrasco, A., Gutiérrez, G. y Flores, C. (2017). Failed regulations and school composition: Selective admission practices in Chilean primary schools. Journal of Education Policy, 32(5), 642-672. https://doi.org/10.1080/02680939.2017.1312549

Castillo, D., Santa Cruz, E. y Vega, A. (2018). Estudiantes migrantes en escuelas públicas chilenas. Calidad en la Educación, 49,18-49. https://doi.org/10.31619/caledu.n49.575

CEM. (2019). Minuta 06. Minuta de variación de matrícula preliminar. Unidad de Apoyo a Gabinete del Centro de Estudios, División de Planificación y Presupuesto, Ministerio de Educación.

CEPAL. (2018). Segundo informe anual sobre el progreso y los desafíos regionales de la agenda 2030 para el desarrollo sostenible en América Latina y el Caribe. CEPAL.

Contreras, D., Sepúlveda, P. y Bustos, S. (2010). When schools are the ones that choose: The effects of screening in Chile. Social Science Quarterly, 91(5), 1349-1368.

https://doi.org/10.1111/j.1540-6237.2010.00735.x

Córdoba, C. (2014). La elección de la escuela en sectores pobres. Resultados de un estudio cualitativo. Psicoperspectivas, 13(1), 56-67.

https://doi.org/10.5027/psicoperspectivas-Vol13-Issue1-fulltext-301

Della Porta, D. (2013). Análisis comparativo: La investigación basada en casos frente a la investigación basada en variables. En D. Della Porta y M. Keating (Eds.), Enfoques y metodologías de las ciencias sociales. Una perspectiva pluralista (pp. 211-236). Akal.

Elacqua, G., Montt, P. y Santos, H. (2013). Evidencias para eliminar gradualmente el financiamiento compartido. Universidad Diego Portales.

Falabella, A., Seppänen, P. y Raczynski, D. (2015). Growing tolerance of pupil selection: Parental discourses and exclusionary practices in Chile and Finlandia. En P. Seppänen, A. Carrasco, M. Kalalahti, R. Rinne y H. Simola (Eds.), Contrasting dynamics in education politics of extremes. School choice in Chile and Finland (pp. 121-138). Sense Publisers.

https://doi.org/10.1163/9789463002622_006

Flores, C. y Carrasco, A. (2013). (Des)igualdad de oportunidades para elegir escuela. Preferencias, libertad de elección y segregación escolar. Espacio Público. https://bit.ly/2NZH2ZW

Godoy, F., Salazar, F. y Treviño, E. (2014). Prácticas de selección en el sistema escolar chileno: Requisitos de postulación y vacios legales. Universidad Diego Portales.

González González, M. T. (2008). Diversidad e inclusión educativa: Algunas reflexiones sobre el liderazgo en el centro escolar. REICE. Revista Iberoamericana sobre Calidad, Eficacia y Cambio en Educación, 6(2), 82-99. 
Gubbins, V. (2013). La experiencia subjetiva del proceso de elección de establecimiento educacional en apoderados de escuelas municipales de la región metropolitana. Estudios Pedagógicos, 39(2), 165-177. https://doi.org/10.4067/S0718-07052013000200011

Gubbins, V. (2014). Estrategias educativas de familias de clase alta. Un estudio exploratorio. Revista Mexicana de Investigación Educativa, 19 (63), 1069-1089.

Hernández, M. y Raczynski, D. (2015). Elección de escuela en Chile: De las dinámicas de distinción y exclusión a la segregación socioeconómica del sistema escolar. Estudios Pedagógicos, 41(2), 127-141. https://doi.org/10.4067/So7 18-07052015000200008

Hastings, J. y Weinstein, J. M. (2008). Information, school choice, and academic achievement: Evidence from two experiments. The Quarterly Journal of Economics, 123(4), 1373-1414.

Madero, C. y Madero, I. (2012). Elección escolar y selección estudiantil en el sistema escolar chileno. ¿Quién elige quién?: El caso de la educación católica. Revista Mexicana de Investigación Educativa, $17(55), 1267-1295$.

Madrid, S. (2016). Diversidad sin diversidad: Los colegios particulares pagados de élite y la formación de la clase dominante en una sociedad de mercado. En J. Corvalán, A. Carrasco, J. E. García Huidobro (Eds.), Mercado escolar y oportunidad educacional. Libertad, diversidad y desigualdad (pp. 269-299). CEPPE.

Murillo, F. J. (2016). Midiendo la segregación escolar en América Latina. Un análisis metodológico utilizando el TERCE. REICE. Revista Iberoamericana Sobre Calidad, Eficacia y Cambio en Educación, 14(4). https://doi.org/10.15366/reice2016.14.4.002

Murillo, F. J. y Martínez-Garrido, C. (2017). Estimación de la magnitud de la segregación escolar en América Latina. Magis, Revista Internacional de Investigación en Educación, 9(19), 11-30. https://doi.org/10.11144/Javeriana.m9-19.emse

Murillo, F. J. y Martínez-Garrido, C. (2019). Una mirada a la investigación educativa en América Latina a partir de sus artículos. REICE. Revista Iberoamericana sobre Calidad, Eficacia y Cambio en Educación, 17(2), 5-25. https://doi.org/10.15366/reice2019.17.2.001

Murillo, F. J., Belavi, G. y Pinilla, L. M. (2018). Segregación escolar público-privada en España. Papers. Revista de Sociología, 103(3), 307-337. https://doi.org/10.5565/rev/papers.2392

Murillo, F. J., Hernández, R., Martínez-Garrido, C. e Hidalgo, N. (2018). Una panorámica de la segregación social de los centros de educación secundaria en Iberoamérica. En F. J. Murillo (Coord.), Avances en democracia y liderazgo distribuido en educación: Actas del II Congreso Internacional de Liderazgo y Mejora de la Educación (pp. 559-564). RILME.

Navarro, L. (2004). La escuela y las condiciones sociales para aprender y enseñar. Equidad social y educación en sectores de pobreza urbana. UNESCO.

OECD. (2019). PISA 2018 results: Where all students can succeed. OECD Publishing. https://doi.org/10.1787/19963777

Parry, T. (1996). Will pursuit of higher quality sacrifice equal opportunity in education. An analysis of the education voucher system in Santiago. Social Science Quarterly, 77(4), 821841 .

Raczynski, D., Salinas, D., De la Fuente, L., Hernández, M. y Lattz, M. (2010). Hacia una estrategia de validación de la escuela pública-municipal: Imaginarios, valoraciones y demandas de las familias. Ministerio de Educación.

Rojas, M.T., Falabella, A. y Leyton, D. (2016). Madres de clase media frente al mercado educativo en Chile: decisiones y dilemas. En J. Corvalán, A. Carrasco y J. E. García Huidobro, Mercado 
escolar y oportunidad educacional. Libertad, diversidad y desigualdad, Colección Estudios en Educación, Centro de Políticas y Prácticas en Educación (pp. 233-267). CEPPE.

Román, M y Murillo, F. J. (2014) Uso de los resultados de las evaluaciones estandarizadas como criterio de elección y selección de escuelas. Revista Iberoamericana de Evaluación Educativa, $7(1), 5-7$.

Ruiz Tagle. J. (2016). La persistencia de la segregación y la desigualdad en barrios socialmente diversos: Un estudio de caso en La Florida, Santiago. EURE, 42(125), 81-108. https://doi.org/10.4067/SO250-71612016000100004

Ruiz Tagle, J. y López, E. (2014). El estudio de la segregación residencial en Santiago: Revisión crítica de algunos problemas metodológicos y conceptuales. EURE, 4O(119), 25-48. https://doi.org/10.4067/SO250-71612014000100002

Sillard, M., Garay, M. y Troncoso, I. (2018). Análisis al nuevo sistema de admisión escolar en Chile: La región de magallanes como experiencia piloto. Calidad en la Educación, 49, 112-136. https://doi.org/10.31619/caledu.n49.578

Stillerman, J. (2016). Educar a niñas y niños de clase media en Santiago: Capital cultural y segregación socioterritorial en la formación de mercados locales de educación. EURE 42(126), 169-186. https://doi.org/10.4067/S0250-71612016000200008

Urrutia, J., López, H., Sabatini, F. y Rasse, A. (2017). Tolerancia a la diversidad y segregación residencial. Una adaptación del modelo de segregación de Schelling con tres grupos sociales. EURE, 43(130), art 5. https://doi.org/10.4067/s0250-71612017000300005

Valenzuela, J.P., Bellei, C. y De los Ríos, D. (2014). Socioeconomic school segregation in a market oriented educational system. The case of Chile. Journal of education Policy, 29(2), 217-241. https://doi.org/10.1080/02680939.2013.806995.

Van Zanten, A. (2003). Middle class parents and social mix in French urban schools: Reproduction and transformation of class relations in education. International Studies in Sociology of Education, 13(2), 107-124. https://doi.org/10.1080/09620210300200106.

Van Zanten, A. (2015). The determinants and dynamics of school choice. A comparative review. En P. Seppänen, A. Carrasco, M. Kalalahti, R. Rinne y H. Simola (Eds.), Contrasting dynamics in education politics of extremes. School choice in Chile and Finland (pp. 3-28). Sense Publishers. https://doi.org/10.1163/9789463002622_002

Vignoli, J. y Rowe, F. (2019). Efectos cambiantes de la migración sobre el crecimiento, la estructura demográfica y la segregación residencial en ciudades grandes. El caso de Santiago, Chile,1977-2017. CEPAL.

Weinstein, J., Muñoz, G. y Rivero, G. (2018). Los directivos escolares como informantes cualificados de las políticas educativas. Sus opiniones bajo el gobierno de Michelle Bachelet en Chile (2014-2017). REICE. Revista Iberoamericana sobre Calidad, Eficacia y Cambio en Educación, 16(3), 5-27. https://doi.org/10.15366/reice2018.16.3.001

\section{Breve CV de los autores}

\section{Claudia Córdoba}

Psicóloga de la Universidad de Chile y Doctora en Sociología de la Educación por la Universidad Complutense de Madrid. Académica del Departamento de Educación de la Facultad de Humanidades de la Universidad de Santiago de Chile. Sus intereses de investigación han estado alrededor de elección de escuela y segregación socioeconómica 
entre establecimientos educativos, habiendo participado también en estudios sobre iniciación profesional docente. Actualmente desarrolla proyectos de investigación centrados en la elección de escuela por parte de familias migrantes y concentración de este alumnado en escuelas de Santiago de Chile. ORCID ID: https://orcid.org/0000-00030793-3420. Email: claudia.cordoba.c@usach.cl

\section{Alonso Laborda}

Antropólogo Social de la Universidad de Chile y Magíster en Psicología Educacional de la Universidad de Chile. Investigador del Centro de Enseñanza Aprendizaje Campus Sur de la Universidad de Chile. Sus líneas de estudio han estado centradas en orientación para el aprendizaje en educación superior y los procesos de elección de escuela en relación con la segregación socioeconómica y a la integración de familias inmigrantes al sistema escolar chileno. ORCID ID: https://orcid.org/0000-0003-3988-4942. Email: alonso.laborda@uchile.cl

\section{Claudia Reyes}

Antropóloga Social de la Universidad de Chile. Tesista en proyecto Fondecyt $\mathrm{N}^{\circ}$ 11130149 "Análisis de la segregación escolar socioeconómica en Enseñanza Básica", contexto en el cual desarrolló la tesis "Representaciones sociales de las familias sobre educación y su incidencia en la elección de escuela". Sistematizadora de información en "Proceso de consulta a expertos sobre propuesta de Bases Curriculares de $3^{\circ}$ y $4^{\circ}$ medio para el futuro en Educación”, organizado por el PNUD y MINEDUC, 2017. Asesora metodológica para Proyecto de Investigación "Educational imaginaries in motion: Toward a Cultural Political Economic understanding of the chilean educational reform", Facultad de Educación, Universidad de Cambridge, 2019. ORCID: https://orcid.org/0000-0002-6295-5019. Email: cpreyes@ug.uchile.cl 